\title{
A Novel COL4A5 Splicing Mutation Causes Skipping of Exon 14 in a Chinese Family with Alport Syndrome
}

\author{
Erzhi Gao ${ }^{a}$ Xi Yang ${ }^{a} \quad$ Nuo Si ${ }^{b} \quad$ Keqiang Liu ${ }^{b} \quad$ Jin-Quan Wang ${ }^{a}$ Zhihong Liu ${ }^{a}$ \\ ${ }^{a}$ National Clinical Research Center of Kidney Diseases, Jinling Hospital, Nanjing University School of Medicine, \\ Nanjing, China; ${ }^{b}$ McKusick-Zhang Center for Genetic Medicine, State Key Laboratory of Medical Molecular \\ Biology, Institute of Basic Medical Sciences, Chinese Academy of Medical Sciences \& Peking Union Medical College, \\ Beijing, China
}

\section{Keywords}

COL4A5 · Splicing mutations · Alport syndrome $\cdot$ Minigene assay

\begin{abstract}
Background: Alport syndrome (AS) is an inherited progressive renal disease caused by mutations in $\mathrm{COL} 4 A 3, \mathrm{COL} 4 A 4$, and $\mathrm{COL} 4 \mathrm{A5}$. Although mutation screening in the genes responsible for AS is typically performed, only a small proportion of patients receive genetic testing in China, and the functional consequences of multiple splicing variants in AS patients have not been investigated. Methods: A family with $X$-linked AS was diagnosed based on family history and pathological findings from a kidney biopsy. Targeted next-generation sequencing was used to identify the causative mutation, and a minigene assay was performed to test the influence of the mutation on splicing. Results: $A$ c.834+2T $>G$ in COL4A5 was identified and shown to co-segregate with AS in the family. The variant is located in the canonical splicing site and is predicted to induce aberrant splicing. Minigene assay using HEK 293T cells indicated the skipping of exon 14 in COL4A5. Conclusions: The novel COL4A5 splicing mutation identified in the current study broadened the genetic spectrum of X-linked AS and further deepened our insight of the disease's molecular mechanism.

(c) 2019 The Author(s)
\end{abstract}

Published by S. Karger AG, Basel

\section{KARGER}

E-Mail karger@karger.com www.karger.com/kdd

\section{C) 2019 The Author(s)} NonCommercial-NoDerivatives 4.0 International License (CC BY-
This article is licensed under the Creative Commons Attribution NC-ND) (http://www.karger.com/Services/OpenAccessLicense). Usage and distribution for commercial purposes as well as any distribution of modified material requires written permission.

\section{Introduction}

Alport syndrome (AS) is a rare genetic kidney disease characterized by hematuria, progressive renal failure, high-tone sensorineural hearing loss, and ocular abnormalities, such as anterior lenticonus, cataracts, and maculopathy [1]. The prevalence of AS in live births has been estimated at about 1:50,000 [2]. According to the United States Renal Data System (USRDS), approximately 3\% of children and $0.2 \%$ of adults with end stage of renal disease (ESRD) have been diagnosed with AS in the United States [3]. In China, it accounts for an estimated $0.8 \%$ of the patients with kidney diseases [4].

AS is caused by mutations in the type IV collagen genes COL4A3, COL4A4, and COL4A5. These genes encode $\alpha 3, \alpha 4$, and $\alpha 5$ collagen chains, which are major structural components of the glomerular basement membrane as well as the basement membranes in the eye and cochlea [5].

Three AS inheritance modes are recognized: X-linked (XLAS), autosomal recessive, and autosomal dominant. It was recently reported that about $80 \%$ of AS patients have the X-linked dominant inheritance pattern due to

Erzhi Gao and Xi Yang contributed equally to this work.

Zhihong Liu

National Clinical Research Center of Kidney Diseases Jinling Hospital, Nanjing University School of Medicine No. 305 Zhongshan East Road, Nanjing 210016 (China) E-Mail liuzhihong@nju.edu.cn 
mutations in COL4A5. A total of $15 \%$ have an autosomal recessive inheritance pattern caused by $C O L 4 A 3$ and COL4A4, and $5 \%$ are autosomal dominant, which is also caused by COL4A3 and COL4A4 [6-8]. Mencarelli et al. [9] described a digenic form of AS in several families. The causative allelic variants may be cis or trans and result in variable clinical features and transmission patterns [9]. In XLAS patients, hemizygous males exhibit more severe symptoms than heterozygous females, and it is estimated that $60 \%$ reach ESRD by age 30 and $90 \%$ by age 40 [10]

A large number of variants in the AS genes (COL4A3, COL4A4, and COL4A5) have been described, and approximately $13-16 \%$ are splicing mutations. Correlation between genotype and phenotype at the molecular level has not been investigated for the vast majority of variants that have been reported to be pathogenic. In addition, the functional consequences of many splice region variants in AS patients have not been investigated.

We report a novel splice region variant of COL4A5 (c.834+2T $>\mathrm{G}$ ) in a Chinese family with AS and results of the minigene assay used to evaluate the splicing aberration.

\section{Materials and Methods}

\section{Subjects}

Clinical data, laboratory tests, and pathological examination were obtained from the National Clinical Research Center of Kidney Diseases at Jinling Hospital, Nanjing University School of Medicine. Genomic DNA was extracted from peripheral blood using the QIAamp DNA Blood MiNi Kit (Qiagen, Hilden, Germany) according to the manufacturer's instructions and quantified on a Nanodrop ND-1000 spectrophotometer (NanoDrop Technologies, Wilmington, DE, USA). All subjects signed an informed consent form allowing anonymous use of their DNA samples and clinical data for research purposes. The study was approved by the ethics committee at Jinling Hospital.

\section{Mutation Identification}

Medical exome sequencing was performed and a subset of genes associated with genetic kidney disease, including COL4A3, COL4A4, and COL4A5, were analyzed to detect the causative mutation. Library preparation, next-generation sequencing, and bioinformatics analysis were performed by Grandomics Biosciences Co. Ltd. (Beijing, China). A sequencing library targeting all coding regions, canonical splice sites, and at least $30 \mathrm{bp}$ of flanking intron sequences were generated with the NimbleGen SeqCap EZ MedExome kit (Roche NimbleGen, Madison, WI, USA) according to the NimbleGen SeqCap EZ Library SR User Guide (version 3.0). High-throughput sequencing was performed on an Illumina HiSeq X-ten sequencing instrument according to the manufacturer's instructions. The BWA alignment algorithm [11] was used to map Raw reads to the human genome reference build hg19. Next, BAM file results were processed by Picard v1.64 (http://broadin- stitute.github.io/picard/) to mark duplicate reads, and GATK v1.6 [12] was used to perform local realignment around short indels and to recalibrate the quality score of each base call. All enrichment performance tables were generated from these BAM files using Picard's Calculate HSMetrics function with default parameters and extracted from VARBANK's data selection sheets. Coverage of individual genes was obtained from VARBANK's graphical gene coverage module, which computes the per-base coverage directly from the BAM file. Reads were aligned to a reference sequence and variants were called.

The Exome Aggregation Consortium (ExAC Version 0.3.1), Genomes 1000 Project was used to filter common single nucleotide polymorphisms. Compared with the East Asian population of ExAC, variants with a frequency less than 0.01 or 0.05 were in genes responsible for dominant or recessive inherited AS, respectively. The pathogenicity of remaining nonsynonymous variants was predicted by SIFT and polyphen2. Synonymous variants and intron variants in 30-bp segments flanking the exons were also analyzed for impacts on splicing by in silico prediction of human splicing finder. Variant validation was performed by direct Sanger sequencing, and segregation analysis of all available family members was also evaluated. Purified PCR products were sequenced using the same primers and PCR conditions in both directions on an Applied Biosystems 3730xl DNA sequencer. CodonCode Aligner Software (CodonCode Aligner Corporation; Centerville, MA, USA) was used to analyze Sanger sequencing results.

\section{Generation of Minigene Constructs}

The splicing variant c. $834+2 \mathrm{~T}>\mathrm{G}$ is located in the region of the splicing donor site of intron 15 . To study the effect of this variant on splicing, we obtained a 1,058-bp fragment, including exon 1416 (containing exon 14, intron 14, exon 15, intron 15 , and exon 16) and part of the upstream and downstream introns of COL4A5, using the following primers, which were directly amplified from a normal subject's genomic DNA: COL4A5_F: 5'-aagaagtgcaggatccACCTGCCTCCCTCTACTCTTAG-3' and COL4A5_R: $5^{\prime}$ - ctttctcctgggatcc ACCCCATTCTCTGTGATGTCC-3' (lowercase letters indicate nucleotides added to the primers to introduce the BamHI restriction site). The PCR product was purified and inserted into the pCAS2 vector through recombination, which had been digested with BamHI. All of the selected clones were sequenced, and the verified clones were referred to as the wild type (pCAS2-COL4A5-WT). After this step, we used site-directed mutagenesis to obtain mutant-type (pCAS2-COL4A5-MT) clones according to the manufacturer's instructions using the following primers: COL4A5-MU-F: $5^{\prime}$-GTGGTCCTCCAGGAAGTACC-3', COL4A5-MU-R: 5'-GGTACTTCCTGGAGGACCAC-3'. Both primers were retained for expression experiments.

\section{Cell Culture and Minigene Splicing Assay}

HEK 293T cells were cultured in Dulbecco's modified Eagle's medium (Union Cell Resource Center) containing 10\% fetal bovine serum (Gibco) and grown at $37^{\circ} \mathrm{C}$ in a $5 \% \mathrm{CO}_{2}$ and $95 \%$ air incubator according to standard procedures. HEK $293 \mathrm{~T}$ cells that had been seeded at $30 \%$ confluence in $60-\mathrm{mm}$ dishes $16 \mathrm{~h}$ prior were transfected with $3 \mu \mathrm{g}$ of pCOL4A5-c.834+2T, pCOL4A5c. $834+2 \mathrm{G}$. Thirty-six hours after transfection, cells were collected and total RNA was extracted using TRIzol (Invitrogen). Then, $2 \mu \mathrm{g}$ of total RNA was reverse transcribed using a reverse transcription system (Takara) according to the manufacturer's instructions. Fol- 
Fig. 1. Results of renal biopsy. a Periodic acid-Schiff staining shows segmental sclerosis in a glomerulus. b, c Immunofluorescence staining shows normal glomerular basement membrane staining for a 3 type IV collagen and segmental absence of glomerular basement membrane staining for a5 type IV collagen. $\mathbf{d}$ Electron microscopy shows irregular thickening and thinning of glomerular basement membrane.
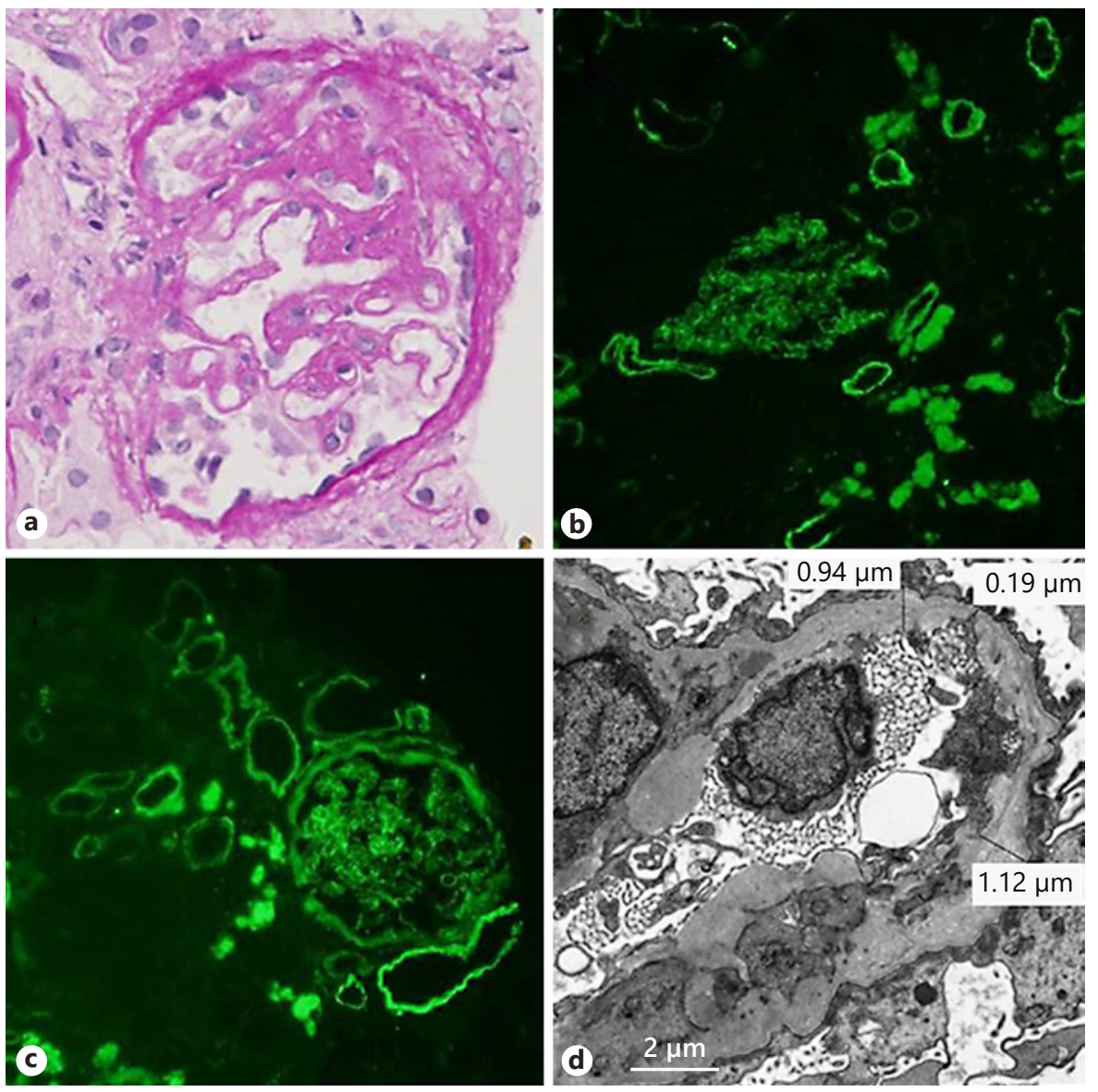

lowing RNA retrotranscription, $200 \mathrm{ng}$ of cDNA from the two constructs were PCR amplified using the primers (reverse transcriptase-PCR-F/R) RTpcr-F: CTGACCCTGCTGACCCTCCT and RTpcr-R: AGGAGGGTCAGCAGGGTCAG.

Agarose gel (2\%) electrophoresis was performed for RT-PCR products obtained from the RNA of HEK 293T cells transfected with the wild-type (WT) or mutant-type (MT) minigene vector.

\section{Results}

\section{Clinical Features and Pedigree Analysis}

The proband (II:2) is a 22-year-old male with history of hematuria, massive proteinuria, and renal failure (chronic kidney disease stage 3 ). He was diagnosed with AS by kidney biopsy (Fig. 1). He does not have high-tone sensorineural hearing loss or ocular abnormalities. The proband's older sister (II:1) only has hematuria, with no proteinuria and normal renal function. The proband's mother (I:2) died in a car accident and had not been tested before her death. The proband's father (I:1) is healthy. Given that III: 1 and III:2 are very young, their parents chose not to have them tested (Table 1). The inheritance mode consists of autosomal recessive or X-linked pattern. The pedigree and Sanger sequencing results are shown in Figure 2.

\section{A Novel Splicing Variant in COL4A5 Causes Exon Skipping}

A c. $834+2 \mathrm{~T}>\mathrm{G}$ variant in the canonical GT-AG splice donor site of COL4A5 was identified by targeted nextgeneration sequencing. No other mutations in COL4A3/ COL4A4/COL4A5 were detected. The variant was not present in the Human Gene Mutation Database and cosegregated with disease phenotype in the family. In silico bioinformatics tools, including Mutation Taster (http:// www.mutationtaster.org) and HSF (http://www.umd.be/ HSF3/), predicted that the $\mathrm{c} .834+2 \mathrm{~T}>\mathrm{G}$ COL4A5 mutations is "disease-causing."

Functional assessment of the WT and c.834+2T>G splice region variant was performed in cultured cells using the pCAS2 splicing reporter minigene assay. WT and MT plasmids were transfected into HEK 293T cells 


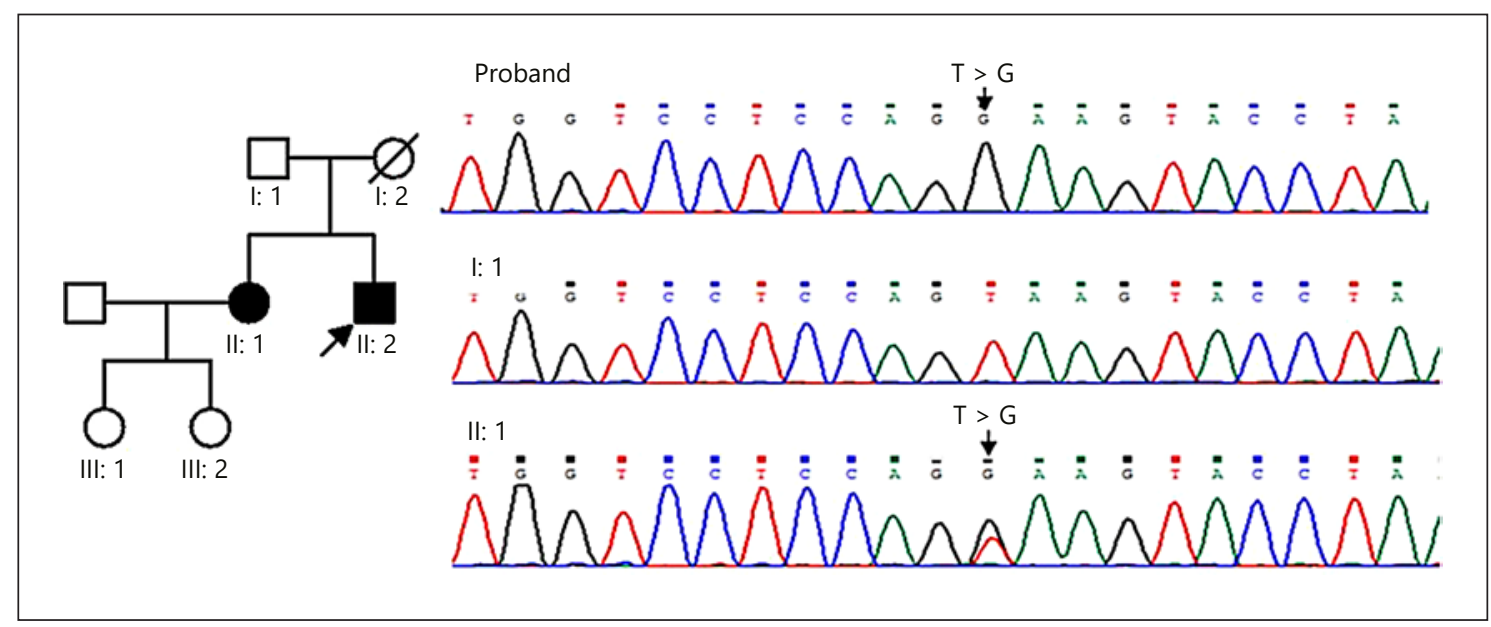

Fig. 2. Pedigree and Sanger sequencing results of a family with Alport syndrome (AS). Filled symbols represent individuals affected with X-linked AS. Normal individuals are denoted by empty symbols. I:2 died in a car accident and had not been tested for urine or blood abnormalities before her death. The patient refuses to have his offspring tested due to their young age.

Table 1. Clinical features of the family with X-linked AS

\begin{tabular}{llllllll}
\hline Patient & Sex & Hematuria & Protein & $\begin{array}{l}\text { Renal } \\
\text { function }\end{array}$ & GBM changes & $\begin{array}{l}\text { Hearing } \\
\text { loss }\end{array}$ & $\begin{array}{c}\text { Ocular } \\
\text { lesions }\end{array}$ \\
\hline I:1 & M & N & N & normal & ND & ND & ND \\
I:2 & F & ND & ND & ND & ND & ND & ND \\
II:1 & F & Y & N & normal & ND & ND \\
II:2 & M & Y & Y & CKD3 & thinning, thickening, & N & N \\
III:1 & F & ND & ND & ND & NDlitting & ND & ND \\
III:2 & F & ND & ND & ND & ND & ND & ND \\
\hline
\end{tabular}

AS, Alport syndrome; CKD, chronic kidney disease; N, no; Y, yes; ND, not detected.

separately. RNA was prepared from the cells and subjected to reverse transcription (RT)-PCR using amplification primers targeting exon A and exon B (Fig. 3a). Agarose gel electrophoresis (Fig. 3b) showed that cells transfected with the WT vector yielded the expected amplicon containing the vector's endogenous exon $\mathrm{A}$, exon $\mathrm{B}$, and COL4A5 exon 14-16, with intronic sequences spliced out. In contrast, cells transfected with the MT vector COL4A5 yielded a major amplicon that lacked exon 14. The sequencing traces presented in Figure $3 \mathrm{c}$ show two different fragments: the 363-bp fragment represents normal splicing with complete exon 14 and the 309-bp fragment represents abnormal splicing, skipping exon 14.

\section{Discussion}

In this study, we identified a novel splicing mutation in COL4A5 (c.834+2T>G) in a Chinese family with AS using targeted gene capture and next-generation sequencing, which was validated by Sanger sequencing. The c. $834+2 \mathrm{~T}>\mathrm{G}$ variation lies in the canonical GT-AG splicing region, and Human Splicing Finder and Mutation Taster software predicted it to most likely affect splicing. The minigene assay identified complete skipping of exon 14 in COL4A5, providing strong evidence to support the pathogenicity of this variant. Massella et al. [13] found a c. $834+2 \mathrm{~T}>\mathrm{C}$ mutation in COL4A5 at the same position reported herein, but with a different nu- 


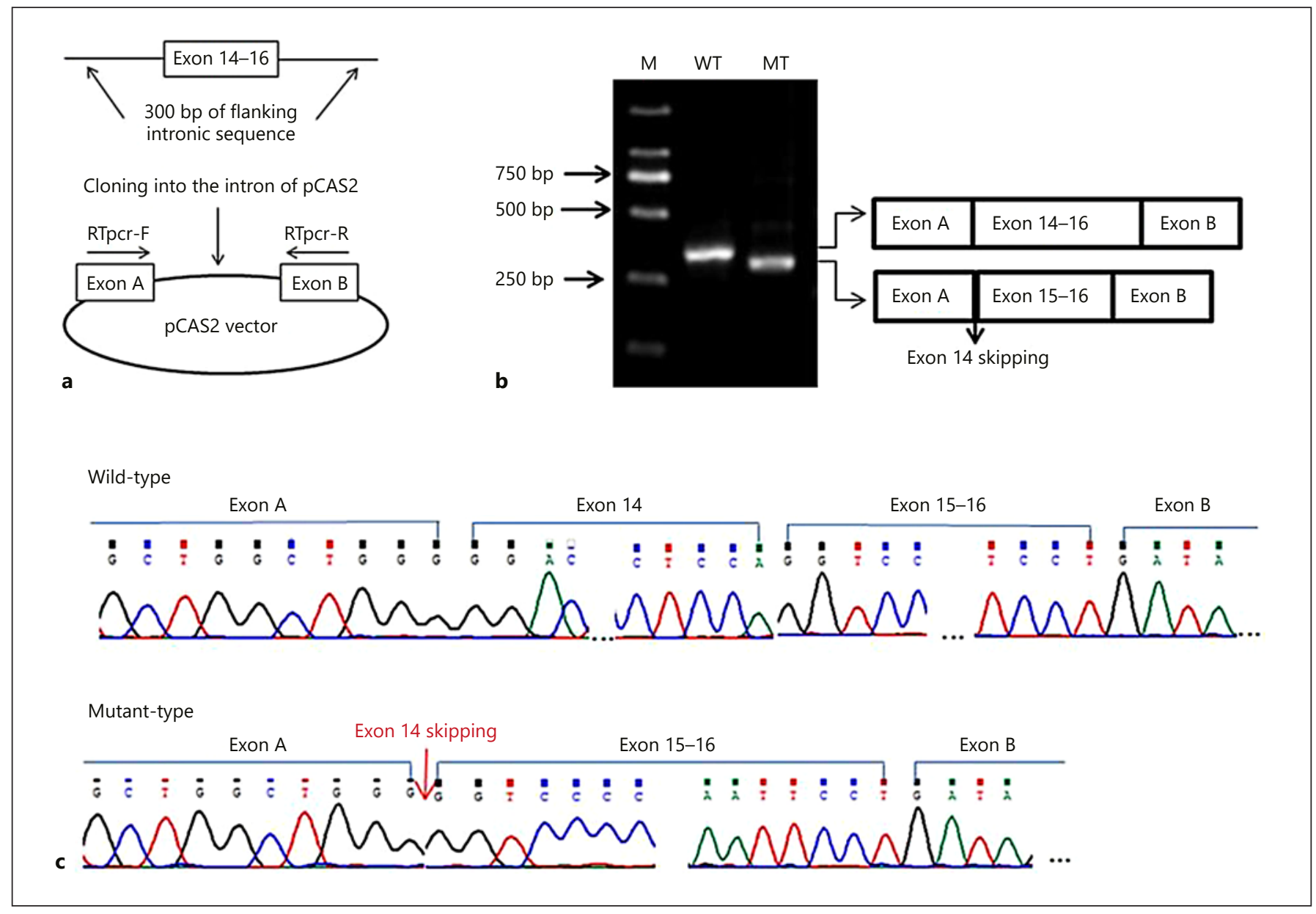

Fig. 3. Impact of the $c .834+2 \mathrm{~T}>\mathrm{G}$ mutation on COL4A5 premRNA splicing. a The wild-type exonic sequences of exon 14-16 of COL4A5 were PCR amplified from the genomic DNA of a healthy subject together with $\sim 300$ bp of their $5^{\prime}$ and $3^{\prime}$ intronic flanking sequences using specific primers. The amplicons were cloned into a pCAS2 reporter vector, which is based on the pcDNA3.1 plasmid and contains a minigene composed of exon A and exon B. b Agarose gel (2\%) electrophoresis of RT-PCR prod- ucts obtained from RNA of HEK 293 cells transfected with the wild-type (WT) or mutant-type (MT) minigene vector. Lanes 2 and 3 represent two independent transfections of the wild-type $($ c. $834+2 \mathrm{~T})$ and mutant-type (c.834+2G) constructs. Lane 1 contains the DL 2000 DNA marker (M). c Sequencing traces of the 363 bp fragment (wild-type) with normal splicing and the $309 \mathrm{bp}$ fragment (mutant-type) in which exon 14 is skipped.

There are six different genes (COL4A1-COL4A6) that encode type IV collagen chains $\alpha 1$ (IV)- $\alpha 6$ (IV). The COL4A5 gene encodes the a 5 chain, which has a carboxyterminal globular NC1 domain (NC1), Gly-Xaa-Yaa repeats in the long central triple-helical domain, and a short amino-terminal 7S domain [5]. In the present study, minigene analysis revealed an aberrant splicing pattern with skipping of exon 14, which led to an in-frame deletion of 18 aa in the Gly-Xaa-Yaa repeat region of the long central triple-helical domain of the a 5 (IV) chain. Thus, the most likely consequence is formation of a truncated a5 (IV) chain with loss of six Gly-Xaa-Yaa structures. As a result, 
the mutation leads to massive proteinuria and chronic kidney disease stage 3 in affected patients.

The rate of AS patients who progress to ESRD varies and is typically influenced by the nature of the COL4A5 pathogenic variant. Large rearrangements and pathogenic frameshift and nonsense variants confer a $90 \%$ probability of ESRD before age 30, with 50\% reaching ESRD by age 20. In individuals with the splicing mutation, the probability of ESRD before age 30 is $70 \%$, with $50 \%$ reaching ESRD by age 25 [10]. Bekheirnia et al. [14] reported that the average age at ESRD onset in males with XLAS is 28,25 , and 37 for those with splice site mutations, truncating mutations, and missense mutations, respectively. That study included 681 males with XLAS from 175 families in the United States. Approximately 12\% of females with XLAS develop ESRD before age 40, increasing to $30 \%$ by age 60 and $40 \%$ by age 80 . This rate could be overestimated because approximately one-third of less severely affected females were lost to follow-up, but it suggests that AS in females is less benign than previously thought [15]. The 21-year-old male proband in the present study had chronic kidney disease stage 3 and is suspected to reach ESRD in 5 years, while his older sister only has hematuria with no proteinuria and normal renal function. A skewed X-inactivation pattern has been shown to be responsible for the phenotypic expression of diseases in women carrier of X-linked disease genes $[16,17]$. The Xchromosome inactivation could be the possible explanation for the milder manifestations of the sister than the proband.

The proband and his sister do not have any extrarenal manifestations, such as high-tone sensorineural hearing loss (SNHL) or ocular abnormalities. SNHL in AS patients is not congenital. Merchant et al. [18] examined temporal bones from nine AS patients and reported that histopathologic findings linked to cochlear involvement in AS are abnormalities in the basement membrane of cells and dysmorphogenesis of the organ of Corti. The authors hypothesized that those abnormalities result in SNHL by altering cochlear micromechanics [18]. SNHL develops in $80-90 \%$ of males with XLAS. Ocular lesions occur in $30-40 \%$ of individuals by age 40 , but may not be detectable in some families until very late in life. Because the proband and his sister are younger than 30 years, they are not likely to have SNHL or ocular lesions. Chen et al. [19] also reported AS in a Chinese family caused by a c.547-3C > A splicing mutation in COL4A5 in which none of the family members had SNHL or ocular abnormalities. We infer that the splice-site variant in this AS family may result in an in-frame deletion of 18 aa, which does not form a premature stop codon or break the reading frame. As such, the proband and his sister do not have any extrarenal manifestations at this age, although they may or may not occur later in life.

There are several limitations to this study. The pedigree is small and only the proband, his sister, and his father have undergone genetic analysis because the mother died in a car accident before her genotype could be tested and the proband's nieces are very young. The proband was diagnosed with AS based on clinical features, pathological findings, and the result of genetic testing. Given that the proband's sister carries the c. $834+2 \mathrm{~T}>\mathrm{G}$ splicing mutation in COL4A5 and her urine is abnormal, but his father does not carry the variant and has normal urine, we hypothesize that this variation segregates with the phenotype. Although the proband's mother died prior to genetic testing, she likely carried the variation. In families with X-linked dominant inheritance, mothers who have a heterozygous pathogenic variant in COL $4 A 5$ have a $50 \%$ chance of transmitting the variant to their children in each pregnancy. Affected males will pass the pathogenic variant to all of their daughters and none of their sons. In the present study, the proband's mother likely transmitted the pathogenic variant to both her son and daughter.

In conclusion, this study identified a novel splicing mutation in COL4A5 in a Chinese family with XLAS. A minigene assay was used to show that this variant is a pathogenic mutation that causes complete skipping of exon 14.

\section{Acknowledgements}

We sincerely thank the patients and their families for assisting us in completing this study. This work was supported by the National Key Research and Development Program of China (2016YFC0904103).

\section{Statement of Ethics}

All subjects signed an informed consent form allowing anonymous use of their DNA samples and clinical data for research purposes. The study was approved by the ethics committee at Jinling Hospital.

\section{Disclosure Statement}

The authors declare that they have no potential conflicts of interest.
Gao/Yang/Si/Liu/Wang/Liu 


\section{References}

1 Tryggvason K, Zhou J, Hostikka SL, Shows TB. Molecular genetics of Alport syndrome. Kidney Int. 1993 Jan;43(1):38-44.

2 Levy M, Feingold J. Estimating prevalence in single-gene kidney diseases progressing to renal failure. Kidney Int. 2000 Sep;58(3):92543.

3 Kashtan CE. Alport syndrome. An inherited disorder of renal, ocular, and cochlear basement membranes. Medicine (Baltimore). 1999 Sep;78(5):338-60.

4 Hou JH, Zhu HX, Zhou ML, Le WB, Zeng $\mathrm{CH}$, Liang SS, et al. Changes in the Spectrum of Kidney Diseases: An Analysis of 40,759 Biopsy-Proven Cases from 2003 to 2014 in China. Kidney Dis (Basel). 2018 Feb;4(1):10-9.

5 Hudson BG. The molecular basis of Goodpasture and Alport syndromes: beacons for the discovery of the collagen IV family. J Am Soc Nephrol. 2004 Oct; 15(10):2514-27.

6 Fallerini C, Dosa L, Tita R, Del Prete D, Feriozzi $S$, Gai G, et al. Unbiased next generation sequencing analysis confirms the existence of autosomal dominant Alport syndrome in a relevant fraction of cases. Clin Genet. 2014 Sep;86(3):252-7.

7 Morinière $\mathrm{V}$, Dahan $\mathrm{K}$, Hilbert $\mathrm{P}$, Lison $\mathrm{M}$, Lebbah S, Topa A, et al. Improving mutation screening in familial hematuric nephropathies through next generation sequencing. J Am Soc Nephrol. 2014 Dec;25(12):2740-51.
8 Hertz JM, Thomassen M, Storey H, Flinter F. Clinical utility gene card for: alport syndrome - update 2014. Eur J Hum Genet. 2015 Sep; 23(9):1269.

9 Mencarelli MA, Heidet L, Storey H, van Geel M, Knebelmann B, Fallerini C, et al. Evidence of digenic inheritance in Alport syndrome. J Med Genet. 2015 Mar;52(3):163-74

10 Jais JP, Knebelmann B, Giatras I, De Marchi M, Rizzoni G, Renieri A, et al. X-linked Alport syndrome: natural history in 195 families and genotype- phenotype correlations in males. J Am Soc Nephrol. 2000 Apr;11(4):649-57.

$11 \mathrm{Li} \mathrm{H}$, Durbin R. Fast and accurate short read alignment with Burrows-Wheeler transform. Bioinformatics. 2009 Jul;25(14): 1754-60.

12 McKenna A, Hanna M, Banks E, Sivachenko A, Cibulskis K, Kernytsky A, et al. The Genome Analysis Toolkit: a MapReduce framework for analyzing next-generation DNA sequencing data. Genome Res. 2010, 20(9): 1297-1303.

13 Massella L, Gangemi C, Giannakakis K, Crisafi A, Faraggiana T, Fallerini C, et al. Prognostic value of glomerular collagen IV immunofluorescence studies in male patients with X-linked Alport syndrome. Clin J Am Soc Nephrol. 2013 May;8(5):749-55.

14 Bekheirnia MR, Reed B, Gregory MC, McFann K, Shamshirsaz AA, Masoumi A, et al. Genotype-phenotype correlation in X-linked Alport syndrome. J Am Soc Nephrol. 2010 May;21(5):876-83.
15 Jais JP, Knebelmann B, Giatras I, De Marchi M, Rizzoni G, Renieri A, et al. X-linked Alport syndrome: natural history and genotype-phenotype correlations in girls and women belonging to 195 families: a "European Community Alport Syndrome Concerted Action" study. J Am Soc Nephrol. 2003 Oct;14(10): 2603-10.

16 Brown RM, Brown GK. X chromosome inactivation and the diagnosis of $\mathrm{X}$ linked disease in females. J Med Genet. 1993 Mar;30(3):17784.

17 Jørgensen AL, Philip J, Raskind WH, Matsushita M, Christensen B, Dreyer V, et al. Different patterns of $\mathrm{X}$ inactivation in MZ twins discordant for red-green color-vision deficiency. Am J Hum Genet. 1992 Aug;51(2): 291-8.

18 Merchant SN, Burgess BJ, Adams JC, Kashtan CE, Gregory MC, Santi PA, et al. Temporal bone histopathology in alport syndrome. Laryngoscope. 2004 Sep;114(9):1609-18.

19 Chen C, Lu CX, Wang Q, Cao LH, Luo Y, Zhang X. A Novel Splicing Mutation Identified in a Chinese Family with X-linked Alport Syndrome Using Targeted Next-Generation Sequencing. Genet Test Mol Biomarkers. 2016 Apr;20(4):203-7. 